\title{
ORBIT DRIFT CORRECTION USING CORRECTORS WITH ULTRA-HIGH DAC RESOLUTION*
}

\author{
R. Bakker, K. Bürkmann, F. Falkenstern, J. Feikes, B. Franksen, R. Görgen, P. Kuske, \\ R. Lange, I. Müller, R. Müller ${ }^{\dagger}$, J. Rahn, T. Schneegans, BESSY, Berlin, Germany
}

\begin{abstract}
At BESSY the planned continous orbit drift correction could not go into routine operation as originally foreseen: the resolution of the $3 \mathrm{mrad}$ correctors controlled by 16 bit DACs was insufficient and perturbed specific experiments unacceptably[1]. Now a novel $2 \times 16$ bit coarse/fine type I/O board solved this problem while preserving the full dynamic range of the correctors. Permanent correction activity no more deteriorates experimental conditions. A typical orbit definition within $+/-5 \mu \mathrm{m}$ at more than $90 \%$ of the BPMs during a day is achieved. Even large perturbations caused by e.g. decaying superconducting wave length shifter currents or residual effects of undulator operations are adequately suppressed.
\end{abstract}

\section{INTRODUCTION}

Despite a typically high intrinsic orbit stability of a third generation light source there are a number of systematic or unpredictable influences that require a continous slow orbit drift control. At BESSY II the installed high end 16 bit DAC control of the corrector power supplies turned out to be too coarse for some experimental resolutions achieved at the very beginning[1]. As a consequence only one scheduled orbit correction per fill was tolerated by some users. With increasing experimental sensitivity and user activity the consequences from this restriction became unacceptable during the course of last year.

Three obvious solutions to reduce the noise induced by the orbit correction have been considered:

- Improved algorithm: Instead of applying standard SVD to the full set of corrector magnets a sophisticated selection of steerer sub-sets as well as a SVD modification taking the boundary conditions of finite resolution into account are both promising approaches. Drawback is the limited margin of improvement and the considerable amount of beam time required for tests.

- Reduced dynamic range of correctors: This measure would disable a number of diagnostic procedures requiring 'large' kicks or bumps. In addition the recalibration of all corrector power supplies include a finite risk for smooth operational conditions.

- I/O DAC resolution enhancement: Subdividing the 'coarse' setpoint steps of the well established 16 bit

\footnotetext{
* Funded by the Bundesministerium für Bildung, Wissenschaft, Forschung und Technologie (BMBF) and the Land Berlin

† mueller@mail.bessy.de
}

\begin{abstract}
DAC with another identical device promises a full range solution. In case of problems the disabling of the second DAC would allow to return to the established configuration without hardware modification.
\end{abstract}

\section{$22 X 16$ BIT I/O BOARD}

A new I/O board has been developed [2] with two identical high precision 16 bit DACs in series. Full 16 bit addressing of the 24 bit driver software is used for fine DAC steps, the upper 8 bit step the coarse DAC. The lower 8 bit of the coarse DAC could be used for static offsets. The output voltage is calibrated for best linear interpolation of the whole output range and not for strict voltage monotony.

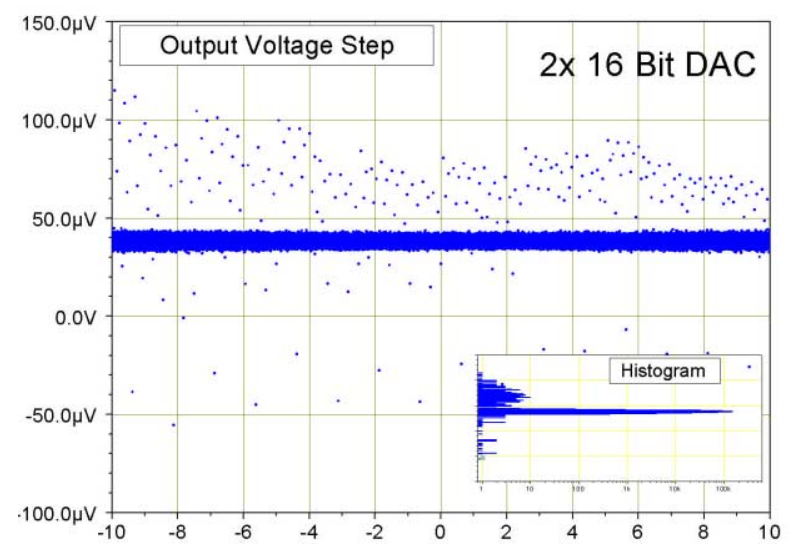

Figure 1: Measured output voltage steps of a $2 \times 16$ bit I/O board operated with a 19 bit resolution.

Nominal resolution of 24 bit is $1.192 \mu \mathrm{V}$ in the bipolar $+/-10 \mathrm{~V}$ mode. Measured noise of the DAC output voltage in the frequency range up to $10 \mathrm{kHz}$ is $17 \mu \mathrm{V}$. In order to be able to estimate the precision improvement to be expected the whole output range has been measured in the laboratory in $2^{19}$ steps of $2^{5}$ digits equivalent to the $38 \mu \mathrm{V}$ clearly above noise threshold (Fig. 1). Most of the measured voltages stay within a $17 \mu \mathrm{V}$ band. Only when the coarse DAC is stepped the noise of this element shows as a large $+/-50 \mu \mathrm{V}$ uncertainty. These results predict that the noise limited resolution improvement of a $19 \mathrm{bit} \mathrm{I} / \mathrm{O}$ (factor of 8 ) will be exceeded in practical applications. 


\section{PERFORMANCE}

Using the 16 bit I/O board the stability of the continous correction depends on the correction target. Aiming at the orbit optimal with respect to the centers of the quadrupoles (beam based alignment measurements, $0.1 \mathrm{~mm}$ minimal RMS deviation) showed less induced noise on the beam than stabilizing the beam to a measured 'golden orbit' $(0$ $\mathrm{mm}$ RMS at the time of measurement). As a first empirical hint on the significance of the improved $2 \times 16$ bit resolution the difference between both targets was no more obvious. Now the (frequently recalibrated) 'golden orbit' became the standard correction target: In this configuration failures of BPM or corrector hardware do not automatically lead to an unevitale orbit jump.

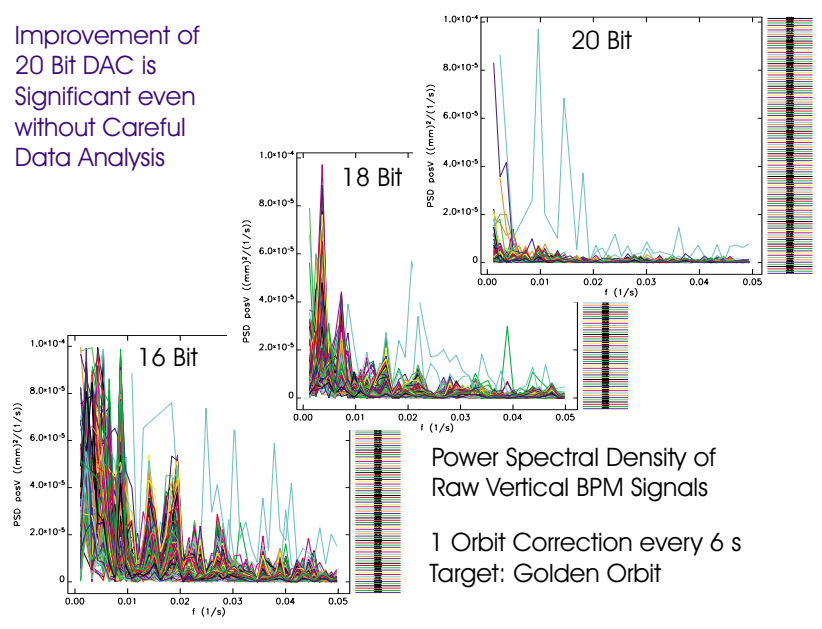

Figure 2: Power spectral density of all 112 raw BPM signals corrected to the 'golden orbit' with 16, 18 and 20 bit resolution. Data aquisition time was of the order of $0.2 \mathrm{~h}$.

As a more quantitative test of the new boards the orbit correction program was configured to artificially reduce the resolution to 16,18 and 20 bit. The BPM signals have been monitored for about 100 corrections. With the pseudo 16 bit resolution the known behaviour is reproduced. As the resolution increases the corrected beam becomes more stable. The power spectral density of these measurements clearly show (see fig. 2) that even the improvement transition from 18 to 20 bit is significant for the beam quality even if this is within the intrinsic noise of the DAC output.

\section{APPLICATIONS}

\subsection{Insertion Device Compensation}

At the beginning of user service gap drives occurred only occasionally and allowed for sufficient time spans of unperturbed operation. Later the once-per-fill correction strategy imposed restrictive requirements on insertion device (ID) transparency: either the internal compensation could suppress residuals below $10 \mu \mathrm{m}$ perturbation or the allowed driving range had been limited accordingly.



Figure 3: Typical BPM signal traces. $90 \%$ of the 112 BPMs stay within a $+/-5 \mu \mathrm{m}$ band. A day of user operation has been selected where shortly after 8:00 am and before 11:00 am the orbit correction has been stopped. In the full scale inset graph the effects of the decaying superconducting WLS field on the horizontal orbit can be seen.

After installation completion of the upgraded corrector $\mathrm{I} / \mathrm{O}$ tests at all high resolution experiments showed that the continous orbit correction no more perturbs but enhances experimental results. The continous drift correction became part of user service. Shortly after that the parallel scan mode of insertion device and monochromator was made available and drive limits have been abandoned. The electro-magnetic undulator U180ID4R has principal limits to improve intrinsic compensation due to hysteresis effects and corrector power supply speed problems. The continous correction allowed for the full drive range in an acceptable slowed down mode opening new experimental flexibility.

\subsection{Super-conducting Wave Length Shifter}

Coincident with the corrector upgrade a $7 \mathrm{~T}$ superconduction wave length shifter (WLS) has been installed and set to $6 \mathrm{~T}$ for the users. The current stabilization of this device caused unacceptable orbit jumps. In persistant current 
mode the field decays with a lifetime of about 2 years. Uncorrected this leads to orbit drifts of up to $1 \mathrm{~mm} / \mathrm{h}$ (fig. 3).

During a fill the orbit correction compensates the decaying field with a large localized bump. At injection the super-conducting field is re-established. In order to fix the energy the adjacent correctors are excluded from the orbit correction boundary condition demanding a minimized horizontal kick average[1].

\section{OUTLOOK}

\subsection{Problems Encountered}

The system typically runs reliable and stable but needs obvious improvements. Since the full potential of the correction scheme is not yet fully exploited the graphical user interface is still attached to the process. So if the graphical server is killed by some reason the correction stops. Failures encountered for the first time still need human interaction. Examples are a sudden persistent offset jump in connection with a precision loss of a single BPM or a processed setpoint not executed by the device. Correction is stopped on plausibility checks to avoid catastrophic orbit discursion and an alarm is fired. Then the correction has to be reestablished by the operator.



Figure 4: Effects of a 1[T] user magnet switched every 5th correction outside the tunnel. The slope is due to the decaying super-conducting field of the WLS.

Some types of orbit perturbations require thorough analysis. Most recent example is an experimental set-up at a dipole beam-line requiring a magnetization reversal magnet outside of the tunnel (fig. 4). Here the adequate countermeasure is shielding or proper cabling and not a modification of the orbit correction procedure.

\subsection{Planned Improvements}

With the continous correction running oscillations at $0.02 \mathrm{~Hz}$ with small amplitude of a few $\mu \mathrm{m}$ are observed by photon BPM monitors[3]. This perturbation is at the resolution limit of the RF BPMs In a pilot experiment it has



Figure 5: Pilot experiment: Residual perturbations seen at the photon BPMs are correctable.

been shown, that a non-oscillating stable beam is achieved feeding back on the photon BPM signals (fig. 5). The idea is now a staged correction scheme: At larger deviations absolute correction to the center of the quadrupoles is done using the RF BPMs. At the resolution limit of the RF BPMs the correction gracefully moves numerical weight on the photon BPM signals. The 'passive' complementary system is used for consistency and hardware sanity checks.

\section{SUMMARY}

From the smooth installation procedure, the minimal number of initial hardware failures and the huge boost in operational performance the upgrade to new $2 \times 16$ bit I/O boards for all correctors in both planes turned out to be an unrestricted success. Now the potential for improvements has moved from the correctors to resolution limits of the RF BPMs. Here data from the photon BPMs will provide a way out.

\section{ACKNOWLEDGEMENT}

Photon beam quality and stability as well as effects of the orbit correction procedures on user experiments have been analysed and documented by the BESSY staff members R. Follath, K. Holldack and F. Senf. Their constructive and constant feedback is gratefully acknowledged.

\section{REFERENCES}

[1] R. Müller at. al., Orbit Control at BESSY II: Present Performance and Plans, EPAC 2000, Vienna, Austria, p. 666

[2] Development has been performed by EuKontroll, Berlin, Germany. Details and contact: EuKontroll@t-online.de

[3] K. Holldack et.al., Review of Emittance and Stability Monitoring Using Synchrotron Radiation Monitors, to be published, DIPAC'01, Grenoble 